\title{
The active quiescence of HR Del (Nova Del 1967)^
}

\section{The ex-nova HR Del}

\author{
P. Selvelli ${ }^{1}$ and M. Friedjung ${ }^{2}$ \\ 1 CNR-IASF-Osservatorio Astronomico di Trieste, Via Tiepolo 11, 34131 Trieste, Italy \\ 2 Institut d'Astrophysique, 98 boulevard Arago, 75014 Paris, France \\ e-mail: fried@iap.fr
}

Received 31 October 2002 / Accepted 17 January 2003

\begin{abstract}
This new UV study of the ex-nova HR Del is based on all of the data obtained with the International Ultraviolet Explorer (IUE) satellite, and includes the important series of spectra taken in 1988 and 1992 that have not been analyzed so far. This has allowed us to make a detailed study of both the long-timescale and the short-timescale UV variations, after the return of the nova, around 1981-1982, to the pre-outburst optical magnitude. After the correction for the reddening $\left(E_{B-V}=0.16\right)$, adopting a distance $d=850$ pc we have derived a mean UV luminosity close to $L_{\mathrm{UV}} \sim 56 L_{\odot}$, the highest value among classical novae in "quiescence". Also the "average" optical absolute magnitude $\left(M_{v}=+2.30\right)$ is indicative of a bright object. The UV continuum luminosity, the HeII $1640 \AA$ emission line luminosity, and the optical absolute magnitude all give a mass accretion rate $\dot{M}$ very close to $1.4 \times 10^{-7} M_{\odot} \mathrm{yr}^{-1}$, if one assumes that the luminosity of the old nova is due to a non-irradiated accretion disk. The UV continuum has declined by a factor less than 1.2 over the 13 years of the IUE observations, while the UV emission lines have faded by larger factors. The continuum distribution is well fitted with either a black body of $33900 \mathrm{~K}$, or a power-law $F_{\lambda} \sim \lambda^{-2.20}$. A comparison with the grid of models of Wade \& Hubeny (1998) indicates a low $M_{1}$ value and a relatively high $\dot{M}$ but the best fittings to the continuum and the line spectrum come from different models. We show that the "quiescent" optical magnitude at $m_{v} \sim 12$ comes from the hot component and not from the companion star. Since most IUE observations correspond to the "quiescent" magnitude at $m_{v} \sim 12$, the same as in the pre-eruption stage, we infer that the pre-nova, for at least 70 years prior to eruption, was also very bright at near the same $L_{\mathrm{UV}}, M_{v}, \dot{M}$, and $T$ values as derived in the present study for the ex-nova. The wind components in the P Cyg profiles of the CIV $1550 \AA$ and NV $1240 \AA$ resonance lines are strong and variable on short timescales, with $v_{\text {edge }}$ up to $-5000 \mathrm{~km} \mathrm{~s}^{-1}$, a remarkably high value. The phenomenology of the short-time variations of the wind indicates the presence of an inhomogeneous outflow. We discuss the nature of the strong UV continuum and wind features and the implications of the presence of a "bright" state a long time before and after outburst on our present knowledge of the pre-nova and post-nova behavior.
\end{abstract}

Key words. stars: novae, cataclysmic variables - ultraviolet - stars: winds, outflows

\section{Introduction}

HR Del = Nova Del 1967 brightened in July 1967 to a magnitude of 5.5 (Alcock 1967) from a pre-nova magnitude near $m_{v} \sim 12$ (Stephenson 1967; Barnes \& Evans 1970; Robinson 1975). The object remained for as long as 5 months near this pre-maximum halt and brightened again in mid Dec. 1967 to reach a maximum peak of $m_{v} \sim 3.5$ (Terzan 1970; Terzan et al. 1974; Bartolini et al. 1969; Mannery 1970). Both the initial rise of only 7 magnitudes and the very long timescale to reach maximum are rather unusual for a nova. The decline after maximum was irregular (in May 1968 the brightness increased again to $m_{v} 4.3$ ) and extremely slow with $t_{3}$ about 225 days (Rafanelli \& Rosino 1978). The beginning of the nebular phase

Send offprint requests to: P. Selvelli, e-mail: selvelli@ts.astro.it

* Based on observations made with the International Ultraviolet Explorer and de-archived from the ESA VILSPA Database. occurred about one year after outburst and the return to the pre-outburst visual magnitude occurred after 1975 (Drechsel et al. 1977; Bruch 1982; Rafanelli \& Rosino 1978) or even after 1981-1982 to judge from the lightcurve of the AAVSO and from the IUE FES counts (see Sect. 4). At the present time the visual magnitude of HR Del shows small oscillations around $m_{v}=12.0$.

Spectroscopic observations made during the first outburst phases revealed expansion velocities in the range from -200 to $-700 \mathrm{~km} \mathrm{~s}^{-1}$ (Hutchings 1968) but values of $-1200 \mathrm{~km} \mathrm{~s}^{-1}$ and up to $-1800 \mathrm{~km} \mathrm{~s}^{-1}$ have been reported during the late decline phases in 1968 (Wallerstein 1968; Rafanelli \& Rosino 1978). Friedjung (1992), from a study of the pre-maximum spectral development, has pointed out the unusual nature of nova HR Del, and suggested that HR Del might only marginally satisfy the conditions for a thermonuclear runaway.

The ejected shell was first observed by Kohoutek (1981) and Solf (1983). Recent observations (Slavin et al. 1994; 


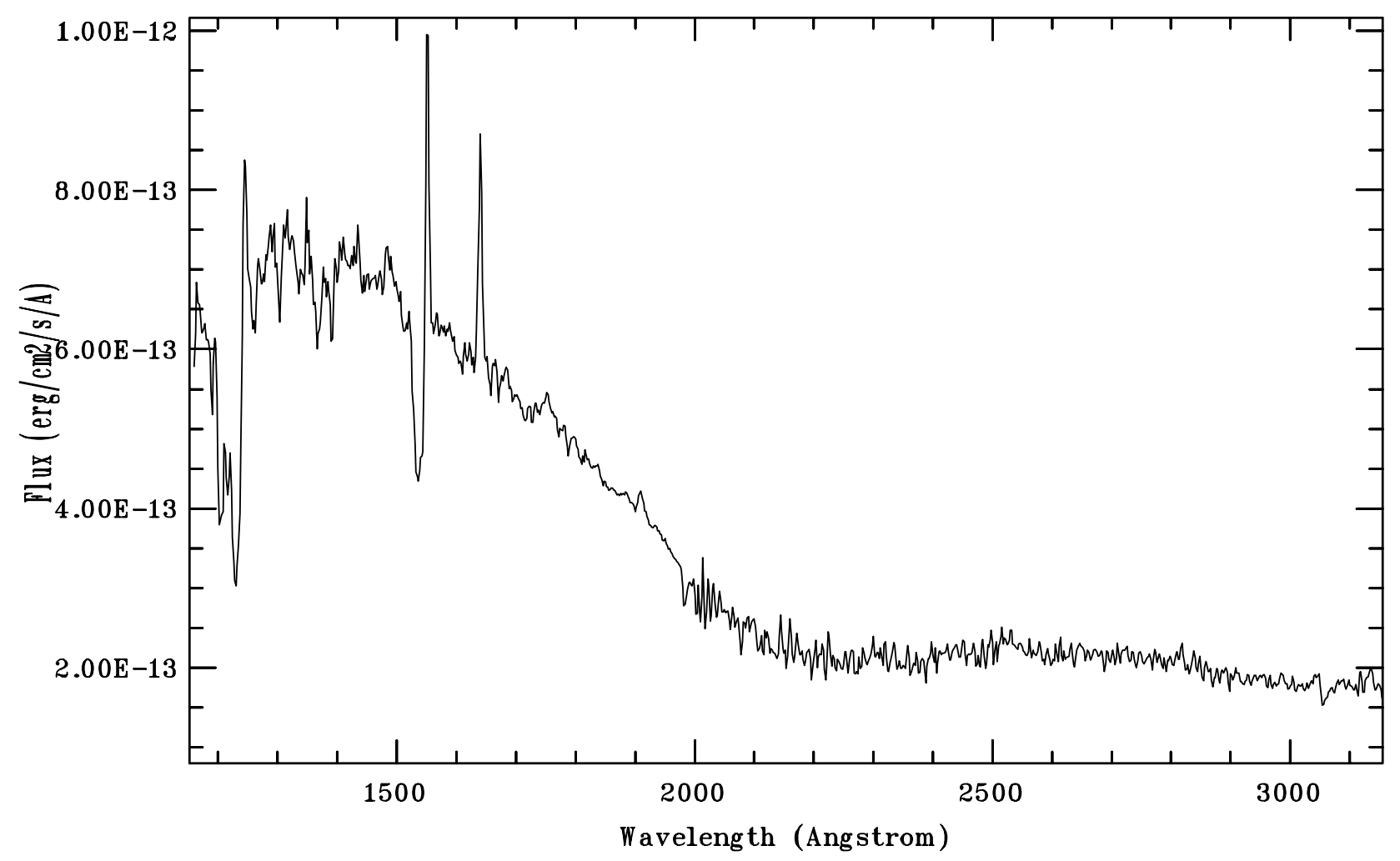

Fig. 1. The "average" 1979-1988 UV spectrum. This "virtual" spectrum has been obtained by averaging and merging all of the SW and LW IUE spectra obtained from 1979 to 1988 . Outstanding features are the P Cyg profiles in the CIV $1550 \AA$ and NV $1240 \AA$ resonance lines. The shortward displaced absorption component of this latter line is blended with an interstellar $\mathrm{Ly}_{\alpha}$ line. Other spectral features are the HeII $1640 \AA$ A emission line and the absorption lines of SiII $1260 \AA$ and CII $1335 \AA$.

Slavin et al. 1995) have revealed a structure with two polar caps and an equatorial ring but there are some discrepancies in the exact shell size (see also Downes \& Duerbeck 2000). Very recently, O'Brien et al. (2002) have reported $v=-290 \mathrm{~km} \mathrm{~s}^{-1}$ and $v=-580 \mathrm{~km} \mathrm{~s}^{-1}$ for the expansion speed in the equatorial ring and in the polar caps respectively and derived a distance $d \sim 1100 \pm 100 \mathrm{pc}$. The position angle of the elongated remnant, as found in different studies, is close to $45^{\circ}$.

The distance, as estimated by several authors using various techniques (expansion parallax, interstellar lines, MMRD, etc.) is near $900 \pm 200 \mathrm{pc}$. The orbital period of the system is 0.214165 and the orbital inclination is close to $40^{\circ}$ (Bruch 1982; Kuerster \& Barwig 1988).

Being one of the brightest nova remnants, HR Del was the target of several IUE observations made in 1979-1980, 1988, and 1992, but the UV literature is based only on the spectra taken in 1979-80 (Krautter et al. 1981; Rosino et al. 1982; Friedjung et al. 1982). These studies all agree upon the presence of a hot continuum and that of strong P Cyg profiles in the CIV $1550 \AA$ lines, but, not surprisingly, a quite wide range of continuum temperatures and outflow velocities have been derived even for the same sets of spectra.

We have undertaken this new analysis of all IUE-INES spectra of HR Del with the purpose of fully exploiting the content of the IUE-INES databank by investigating both the long timescale variation (over more than a decade) and the short timescale variations (over a few hours) in its UV spectrum.

\section{The IUE data}

From the IUE-INES archive we have retrieved both the resampled image (SILO) and the extracted spectrum (MXLO) for the whole set of 49 spectra of HR Del. For a description of the IUE-INES system see Rodriguez-Pascual et al. (1999). Inspection of the SILO images has revealed a good centering in all spectra, and the absence of geo-coronal $\mathrm{Ly}_{\alpha}$ emission, except for a few spectra around SWP07108. All SILO images have been carefully examined for the presence of spurious emission features, blemishes, etc. In the full set of $49 \mathrm{spec}-$ tra, four SWP and two LWR spectra were taken with the small aperture and are of limited use as far as absolute quantities such as continuum and line emission intensities are involved. Also, one spectrum (SWP05757) is badly overexposed in most of it and has been excluded from the sample. Of the remaining 42 spectra ( $31 \mathrm{SWP}+11 \mathrm{LW})$ the most interesting ones are the 23 SWP spectra belonging to the three series of spectra of Aug. 21, 1980, Apr. 29, 1988 and Aug. 30, 1992 since, being obtained in a close sequence over a time baseline slightly longer than one orbital period, they allow a detailed study of the short-timescale variations.

We recall that the IUE data extraction and calibration methods have undergone several revisions during and after the IUE lifetime and that this has resulted in non negligible changes both in the quality of the line spectrum and in shape of the continuum curve (see Gonzalez-Riestra et al. 2001). 


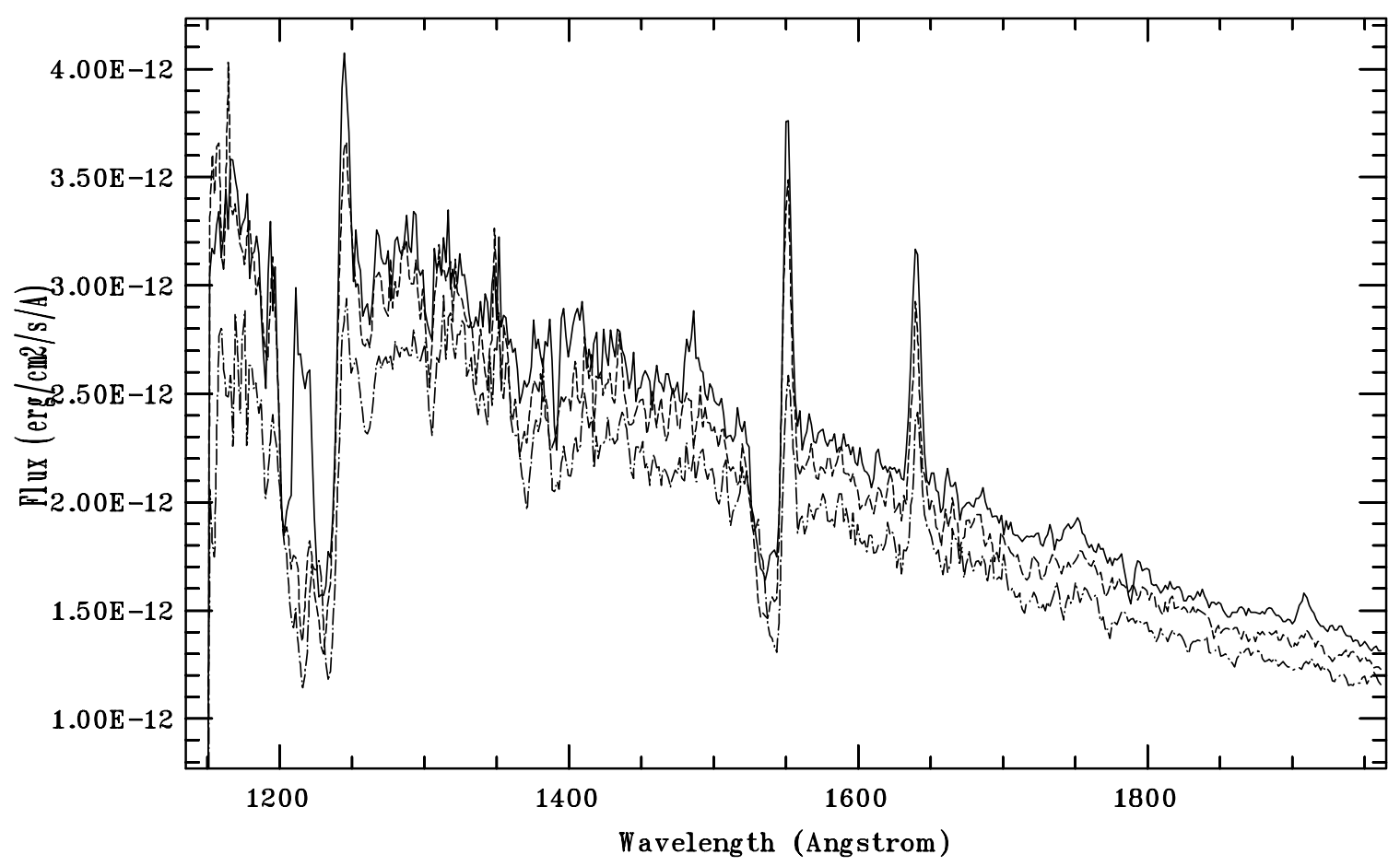

Fig. 2. The long-timescale variations in the SWP range. The "average" SWP spectra of 1980 (top, continuous line), 1988 (mid, dash line), and 1992 (bottom, dot-dash line) are displayed on an absolute scale. The spectra are corrected for reddening with $E_{B-V}=0.16$.

\section{The spectrum and the reddening}

The individual spectra are quite similar to each other in the continuum and line features. This justifies the creation of a "virtual average" spectrum by co-adding and merging all SW and LW spectra for the epochs $(1979,1980,1988)$ in which data for both spectral ranges are available (Fig. 1). In 1992 only SWP data were taken and have not been included in this "average". The improved $S / N$ in the "average" spectrum has allowed both the detection of weak line features and an accurate determination of the reddening : $E_{B-V}=0.16 \pm 0.02$, as estimated by applying the common method of removing the $2175 \AA$ bump. In the present study we will adopt $A_{v}=0.315 \times 0.16 \sim 0.50$.

Outstanding spectral features in most of the UV spectra of HR Del are the strong P Cyg profiles in the CIV $1550 \AA$ and NV $1240 \AA$ A resonance lines, together with the HeII $1640 \AA$ (pure) emission line. The emission component in the $\mathrm{P}$ Cyg profile of the NV $1240 \AA$ line has significantly faded in the most recent spectra (1992). Nebular lines (i.e., NIV $1483 \AA$, OIII $1666 \AA$, NIII $1750 \AA$, and CIII $1909 \AA$ ) are clearly present only in the spectra taken in 1979-1980. The absorption features that are present below $\lambda 1400 \AA$ are identified as SilI $1190 \AA$, SiII $1260 \AA$, OI+SiII $1303 \AA$, CII $1335 \AA$, all these lines being indicative of the spectrum of a B2-B7 star (cf. Rountree \& Sonneborn 1993), and possibly OV $1370 \AA$, from a higher ionization stage.

An interstellar contribution to the zero-volt component in some of these lines cannot be excluded but the presence of intrinsic variations in individual spectra (especially for the SiII $1260 \AA$ line) and the absence of any absorption near MgII $2800 \AA$ indicate that it is quite negligible. An examination of the individual spectra does not reveal substantial changes from spectrum to spectrum with the exception of the shorttimescale variations in the CIV and NV absorption components that are described in Sect. 5. The LW region is almost featureless.

\section{The long-timescale variations}

Figure 2 is a plot (on an absolute scale) of the average SWP spectra for the 1980, 1988 and 1992 epochs that indicates an almost "gray" decay with time in the continuum together with a more pronounced decline in the emission line intensities, especially NV $1240 \AA$ A, CIV 1550 Åand HeII $1640 \AA$. The short wavelength continuum (SWP region) has declined by a factor $\sim 1.19$ from 1979-1980 to 1992 (about 1.08 from 1980 to 1988 ) but the emission line decline is definitely larger: NV $1240 \AA$ is down by $\sim 6$, CIV $1550 \AA$ by $\sim 2.3$ and He II $1640 \AA$ by $~ 1.6$. All nebular lines i.e. NIV $1484 \AA$, NIII $1750 \AA$, and CIII $1909 \AA$ that were clearly present in the 1980 spectra, have declined strongly afterwards.

The pronounced fading in the high ionization emission lines, especially NV $1240 \AA$, together with the moderate decline of both the UV continuum and the optical magnitude over the 13 years covered by the IUE observations would suggest a decline of a very hot, compact source, likely to be associated with post-outburst phenomena, combined with recombination in the nebula.

It is worth mentioning that at the epochs of the first set of IUE observations the V mag was at about 11.9 and declined to 12.0 around 1982. Since then the star has remained at $m_{v} \sim 12.0$, despite the (small) decline in the ultraviolet 


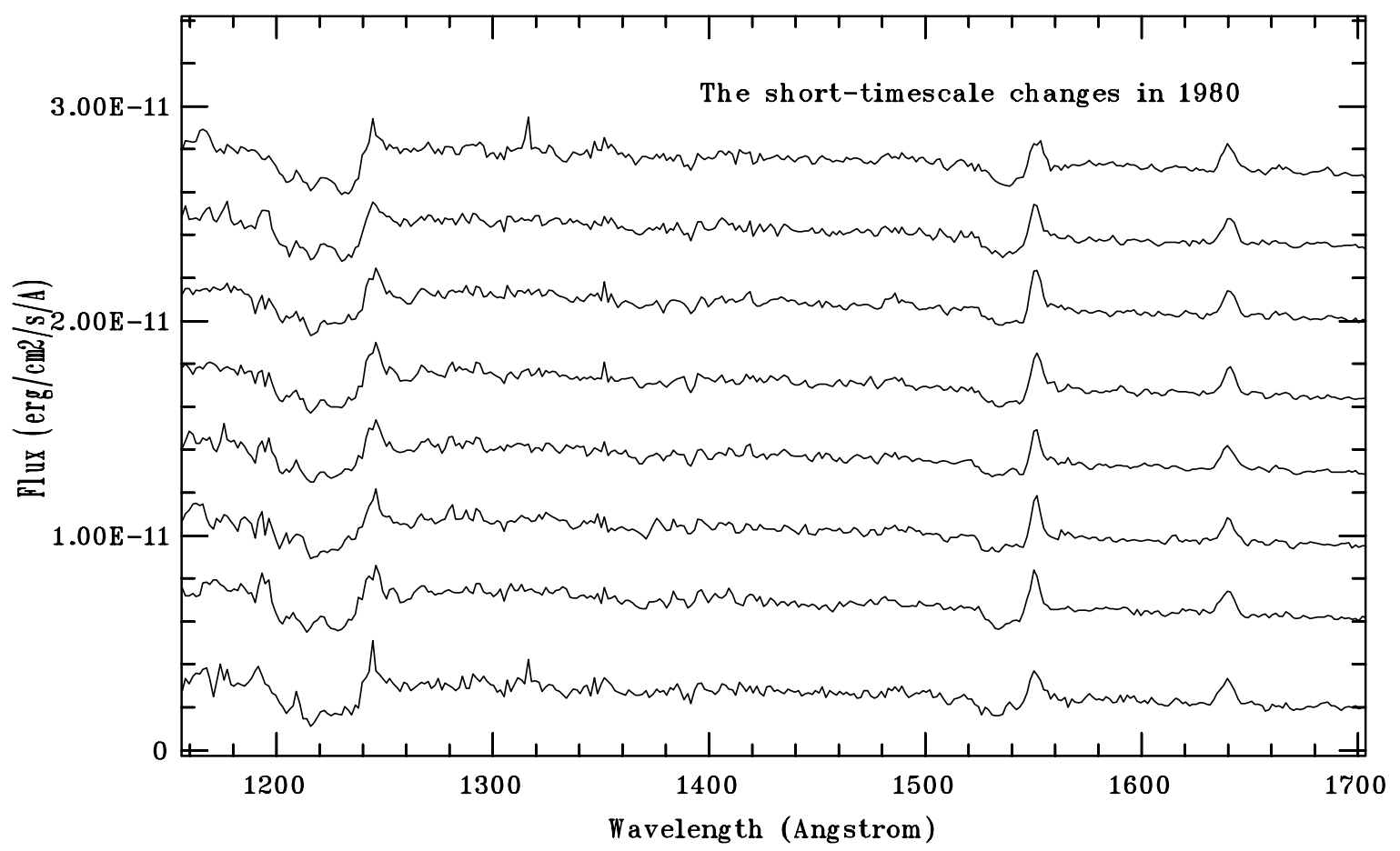

Fig. 3. Display of the short-timescale variations in the spectra of the sequence of Aug. 21, 1980. The spectra have been shifted vertically for display purposes. The spectra are corrected for reddening with $E_{B-V}=0.16$. The average time separation between successive exposures is near $49 \mathrm{~min}$.

region. V optical magnitudes simultaneous with the IUE observations have been obtained from the counts of the FES onboard the IUE satellite, using the calibrations of Perez (1991) and Fireman \& Imhoff (1989).

\section{The short-timescale variations}

The fortunate circumstance that for the 1980, 1988 and 1992 epochs several SWP spectra were taken in a strict sequence of exposures, each sequence covering more than one orbital period ( $\mathrm{P}^{\text {orb }}=5^{\mathrm{h}} 6^{\mathrm{m}} 24^{\mathrm{s}}$, Bruch 1982), it enabled us to study the short-timescale spectral variations and their possible correlation with the orbital phase. Figures 3, 4, and 5 are display plots, on a convenient scale, of the individual spectra taken in the three sequences of 1980, 1988, and 1992.

The short timescale variations do not show any convincing relation with the orbital phase. In a few cases the fluctuations in the observed quantities appear as semi-regular but in most cases they appear as irregular and are probably associated with transient phenomena. Irregular variations in the CIV $1550 \AA$ absorption component occur with timescales of the order of the time separation between successive exposures ( $\sim 50 \mathrm{~min})$, but it is likely that this is just an upper limit set by the observations. Figure 6 is an example of the considerable variations in the absorption component of the CIV $1550 \AA$ doublet. The two spectra (SWP33398 and SWP33402) belong to the 1988 sequence and were taken with time separation of $3^{\mathrm{h}} 53^{\mathrm{m}}$ between the start of the two exposures. Similar variations have been observed also in spectra of 1980 and 1992, although with different time separations. The similar trend and pattern in the changes in the NV and CIV absorption components indicates that the two line forming regions are close or somehow associated.

We have looked for possible overall correlations between quantities such as the emission intensities of the CIV $1550 \AA$ and HeII $1640 \AA$ emission lines, the equivalent width of the absorption component of the CIV $1550 \AA$ line, the far-UV integrated continuum flux, etc. A definite linear trend is present between the intensity of the far-UV-integrated continuum and the emission intensity of the HeII $1640 \AA$ line, while there is evidence of a non-linear correlation between the intensity of the far-UV-integrated continuum and that of the emission intensity of the CIV $1550 \AA$ line (see Fig. 7).

It is remarkable that the intensities of the emission and absorption components in the P Cyg profile of the CIV $1550 \AA$ line are very poorly correlated.

\section{Discussion}

\subsection{The distance to HR Del and the system parameters}

An estimate of the basic system parameters such as the distance $d, M_{1}$, and $R_{1}$ is necessary before any attempt at deter$\operatorname{mining} L_{\mathrm{UV}}, M_{v}, \dot{M}$, etc.

We recall that various methods have yielded a distance to HR Del in the range 700-1100 pc. As an independent check, we add here one more estimate based on the elementary assumption that for this extra slow nova $L \sim L_{\text {Edd }}$ near the outburst maximum. If $M_{1}=0.65 M_{\odot}$ (see the following) then $L_{\text {Edd }}=8.45 \times 10^{37} \mathrm{erg} \mathrm{cm}^{-2} \mathrm{~s}^{-1}$ and $M_{\mathrm{bol}}^{\max }=-6.10$. Near maximum light novae radiate mostly in the optical and the 


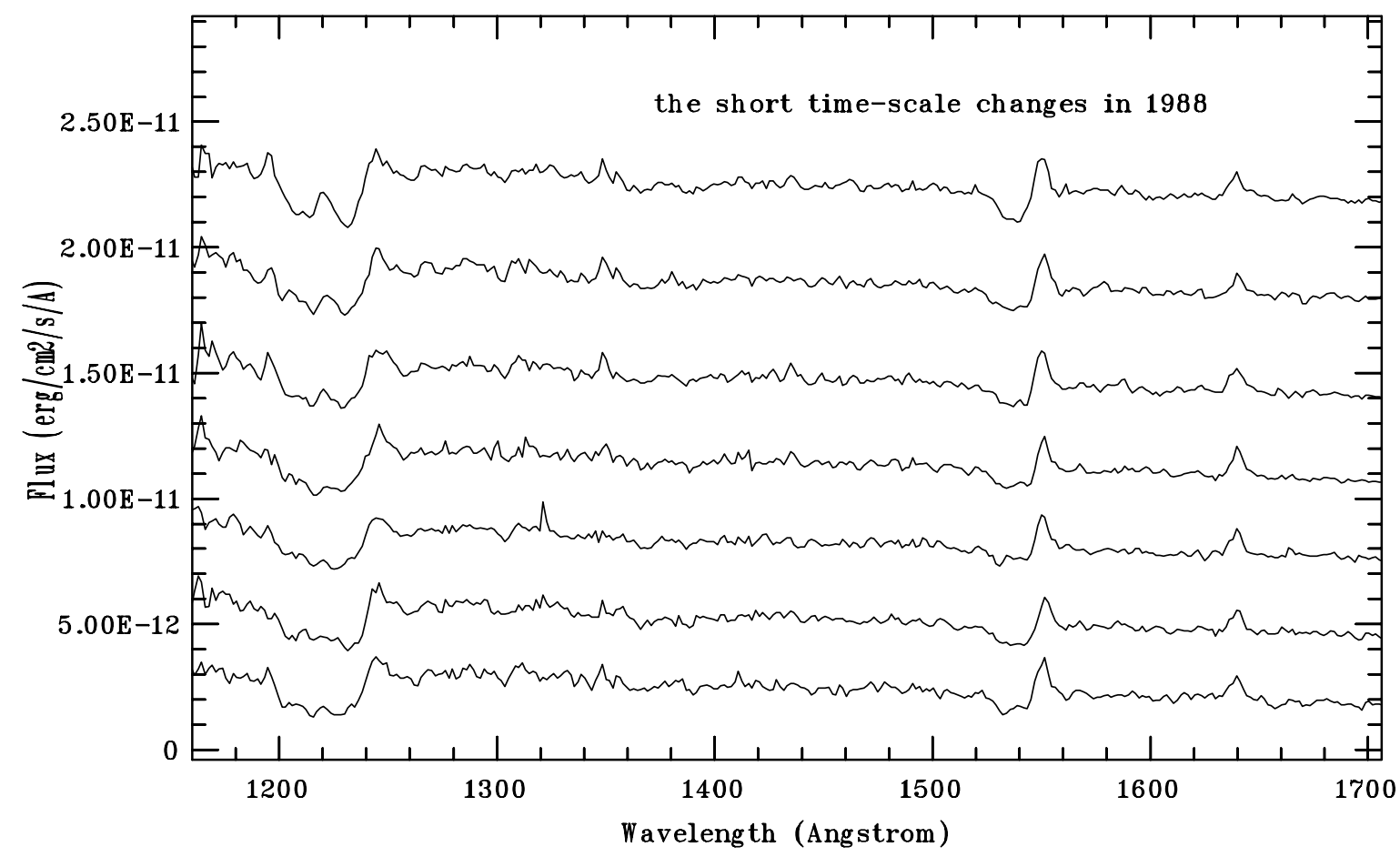

Fig. 4. The same as in Fig. 3 for the sequence of Apr. 29, 1988. The average time separation is near 59 min.

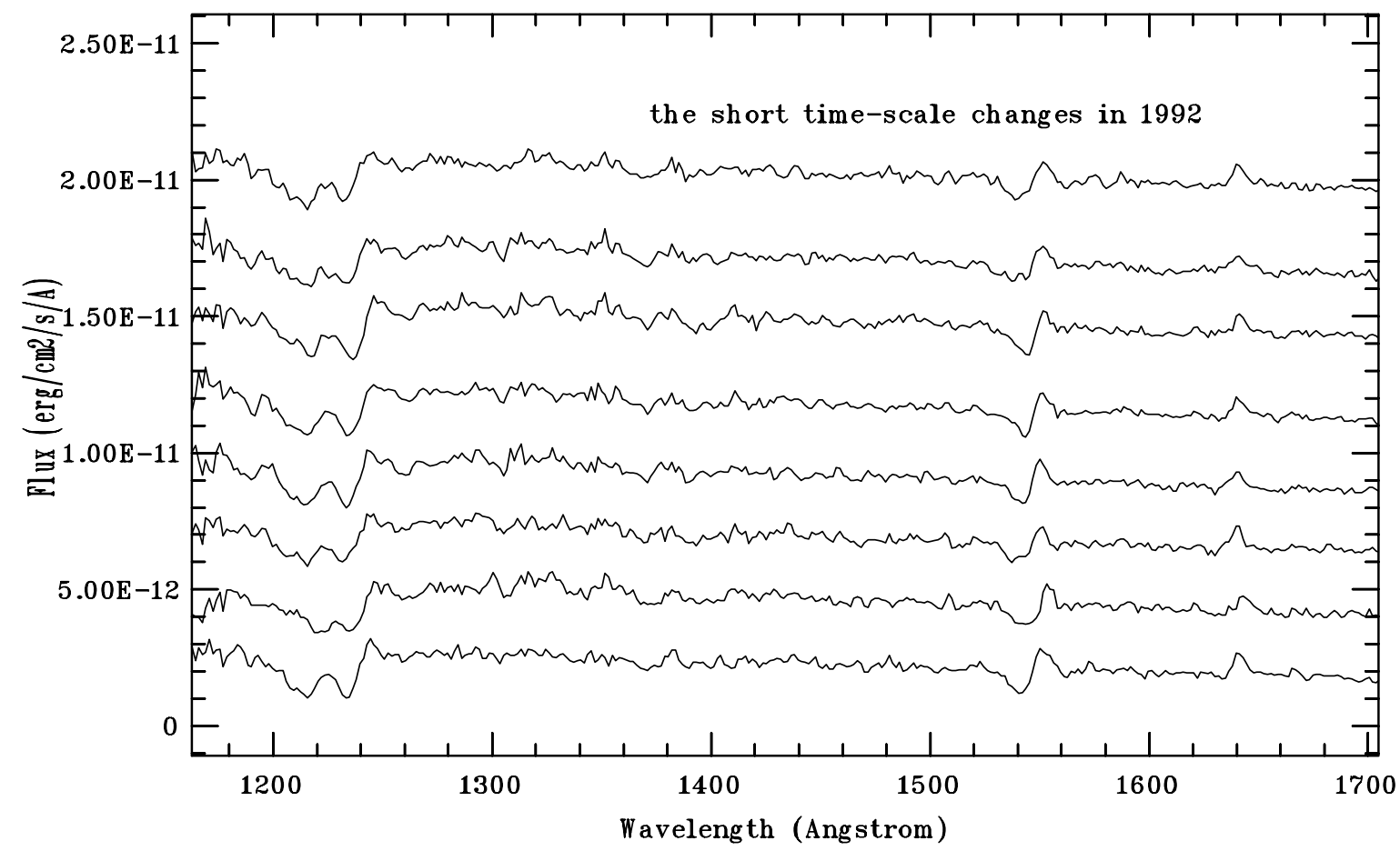

Fig. 5. The same as in Fig. 3 for the sequence of Aug. 30, 1992. The average time separation is near 52 min.

bolometric correction $B C$ is quite small and close to -0.20 . We can therefore confidently assume $M_{v}^{\max } \sim-5.9$. The visual magnitude at maximum $m_{v}^{\max }$ is not clearly defined but is in the range 3.5-5.0. Probably HR Del was slightly under-Eddington in the extended pre-maximum plateau phase at $m_{v}=5.0$, while was slightly super-Eddington (Seitter 1990) in the sharp peak at $m_{v}=3.5$ of December 1967. In this connection let us note that according to Friedjung (1992) HR Del had probably an optically thin wind before the sharp peak and that a superEddington luminosity is not required to accelerate such a wind by radiation pressure. If we assume as $m_{v}^{\max }$ the intermediate value at $m_{v}=4.25$, together with $A_{v}=0.5$, we actually obtain $d=850 \mathrm{pc}$, thus confirming the previous estimates based on other methods. The MMRD relation of Della Valle $\&$ Livio (1995) gives $M_{v}^{\max }=-6.86$ and $d=1180 \mathrm{pc}$ if we take $m_{v}^{\max }=4.0$. The same relation as redetermined in the 


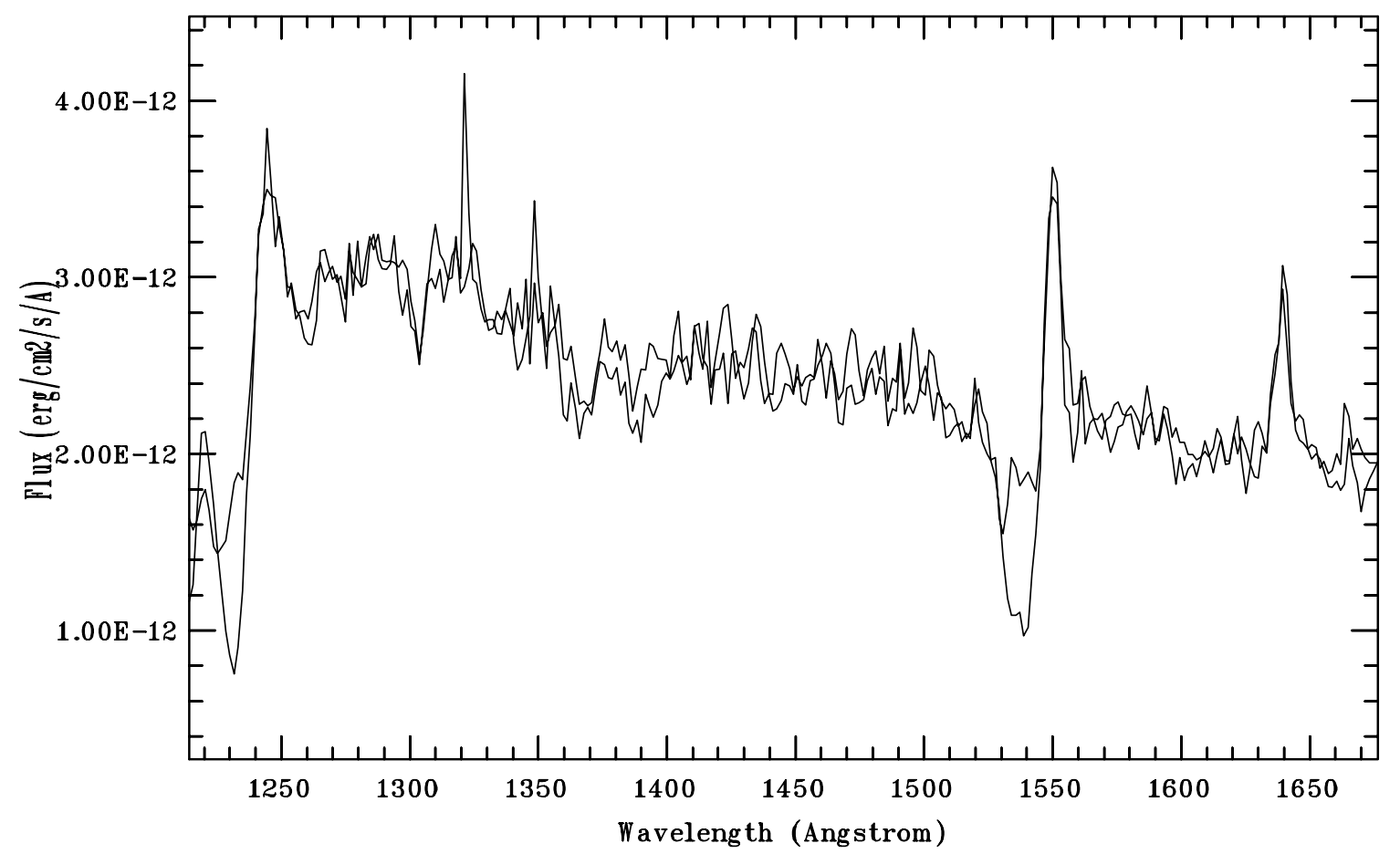

Fig. 6. The dramatic short-timescale changes in the absorption components of the CIV $1550 \AA$ And NV $1240 \AA$ Alnes in two spectra (SWP33398 and SWP33402) of the sequence of April 29, 1988. The changes are remarkably similar in both lines The time separation between the start of the two exposures is of $3^{\mathrm{h}} 53^{\mathrm{m}}$.

zero-point and amplitude by Downes \& Duerbeck (2000) gives instead $M_{v}^{\max }=-6.42$ and $d=960 \mathrm{pc}$. The MMRD of Downes $\&$ Duerbeck (2000) gives $M_{v}^{\max }=-5.99$ and $d=887$ pc. It must be pointed out that there is some degree of uncertainty on the correct value for $t_{2}$ that could affect all these estimates. In the present study we will adopt a rather conservative value for the distance, that is $d=850 \mathrm{pc}$.

$M_{1}$ has been estimated from the mass function

$\frac{\left(M_{2} \cdot \sin i\right)^{3}}{\left(M_{1}+M_{2}\right)^{2}}=1.037 \times 10^{-7} \cdot K_{1}^{3} \cdot P$

adopting $P=0.214165, K_{1}=110 \mathrm{~km} \mathrm{~s}^{-1}$ (Kuerster \& Barwig 1988), $M_{2}=0.53 \pm 0.03 M_{\odot}$ (as the average of recent Mass-Period relationships for CVs reported by Warner 1995; Patterson 1998; Clemens et al. 1998; Smith \& Dhillon 1998), and $i \sim 40^{\circ}$ (as given by Kuerster \& Barwig 1988, reported in Ritter \& Kolb 1998 and supported by the studies on the structure of the nebular shell, as mentioned in Sect. 1). We have allowed a limited variation in both $M_{2}$ and $i$, but, if we constrain $M_{1}$ to values larger than $0.5 M_{\odot}$, then the solutions are within a small interval: for an inclination $i$ lower than $\sim 40^{\circ} M_{1}$ becomes smaller than $M_{2}$, and an $M_{2}$ value as large as $0.60 M_{\odot}$ is required, which seems at the limit of the acceptable $M_{2}$ range. On the other hand an inclination much larger than $i \sim 40^{\circ}$ seems unlikely from the studies on the nova shell. Also, the presence of absorption lines in the spectrum, the high $L_{\mathrm{UV}}$, and the very high outflow velocity (see Sect. 6.7) all point to a medium-low inclination.

With the physical constraints given above, we obtain that the most likely values are: $M_{2}=0.53 \pm 0.03 M_{\odot}$ and $M_{1}$ in the range $0.55 M_{\odot}-0.75 M_{\odot}$ for $i$ in the range $40^{\circ}-45^{\circ}$. In the following we will assume $M_{1}=0.65 \pm 0.10 M_{\odot}$.

We recall that the mean white dwarf (WD) mass $M_{1}$ in classical nova systems, as estimated by nova frequency, is $\sim 1.1 M_{\odot}$ (Ritter et al. 1991), while that estimated from the observations (Smith \& Dhillon 1998) is $0.85 \pm 0.05 M_{\odot}$. Thus, the white dwarf in HR Del seems undermassive. Two pieces of circumstantial evidence, that is, the very slow speed class during outburst $\left(t_{3} \sim 230 \mathrm{~d}\right)$ and the fact that the ejected shell was quite massive, about $10^{-4} M_{\odot}$ (Anderson \& Gallagher 1977) are in agreement with the theoretical expectations for an outburst on a low-mass WD (Livio 1993). If $M_{1} \sim 0.65 M_{\odot}$ we obtain $R_{1}=0.0125 R_{\odot}$, as the average from various $M-R$ relations in white dwarfs as reported by Nauenberg (1972), Paczynski (cf. Anderson 1988), Eggleton (cf. Politano et al. 1990), that do not differ much from each other near $M=0.65 M_{\odot}$.

\subsection{The comparison with model accretion disk spectra}

Wade \& Hubeny (1998) have recently presented a large grid of computed spectra from steady state accretion disks (AD) in luminous CVs. Disk spectra corresponding to twenty-six different combinations of accretion rates and WD masses are computed and tabulated for six different disk inclination angles $i$. The wavelength coverage of the models ranges from $\lambda 800 \AA$ to $\lambda 1200 \AA$, and therefore it is possible to compare them with the IUE spectra taken with the SWP camera ( $\lambda \lambda 1160-1960 \AA)$. It should be pointed out that some notable features in the spectrum of HR Del, e.g. the emission lines and the P Cyg profiles that are probably formed in regions separate from the $\mathrm{AD}$ 


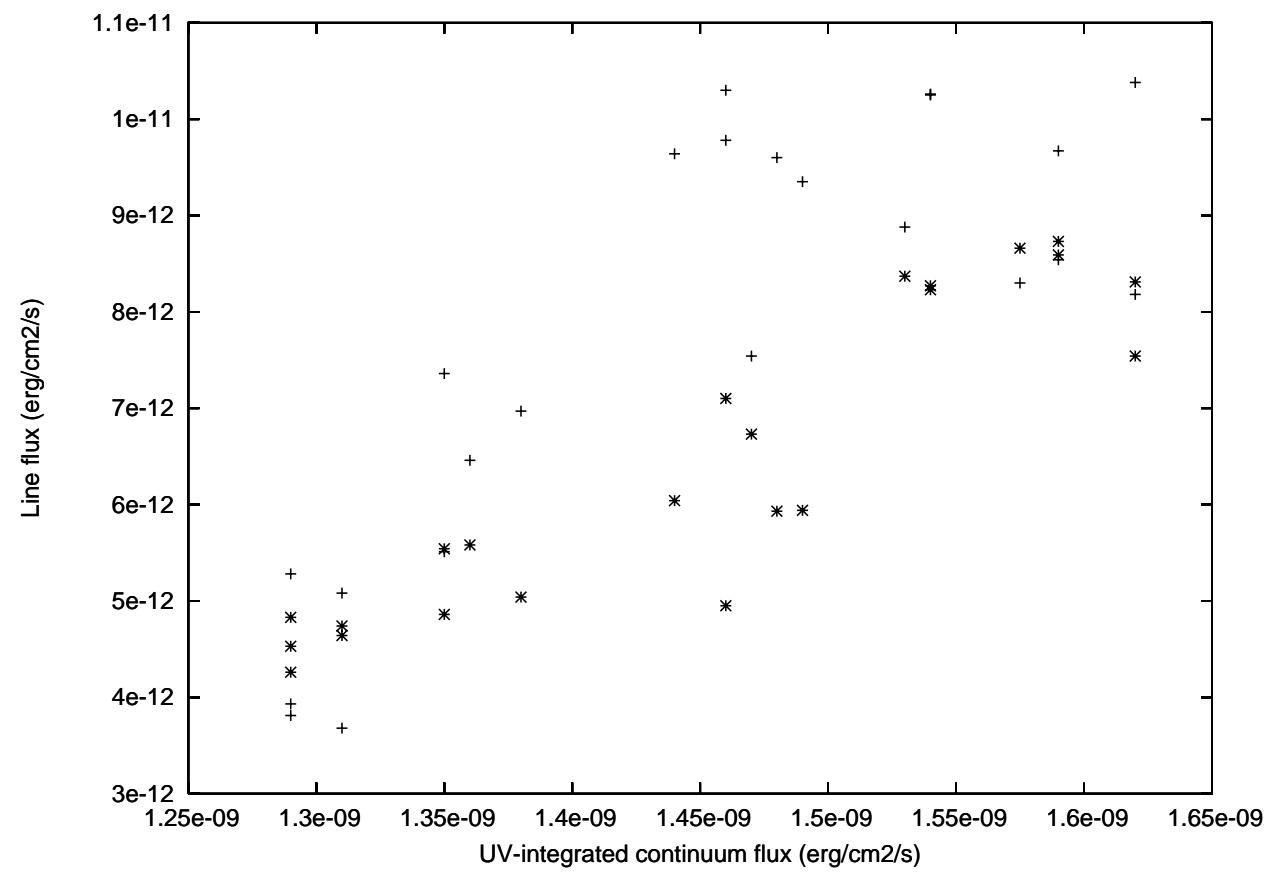

Fig. 7. The overall correlation between the far-UV-integrated continuum flux and the line flux in the CIV $1550 \AA$ (plus symbols) and HeII $1640 \AA$ (asterisks symbols) emission lines.

itself (chromosphere-corona, thick wind), are not present in the model. Therefore, the spectral features we have used for the fitting are the shape of the continuum distribution and the intensity and width of the un-displaced absorption lines (e.g. SiII $1260 \AA$, OI + SiII $1305 \AA$, CII $1335 \AA$, etc).

We have compared most of the 156 different models with the reddening corrected average IUE-SWP spectrum of 19791980 (the spectra at the other epochs differ just in the y-scale because of the near gray decay). The results are only partially satisfactory:

1. The observed continuum flux, when scaled to the normalized distance of the models $(100 \mathrm{pc})$ is higher than in any model. Only model $j j$ (a massive WD at high $\dot{M}$ ) gives fluxes that are comparable with the observed one. Therefore, as far as the continuum is concerned, we have limited the comparison to the slope only.

2. No one solution is convincingly valid at the same time both for the continuum (slope) and the depth and shape of the absorption lines .

3. The best solutions for the continuum (e.g. models $f f$ with $i=75.5^{\circ}$ or $i=41.4^{\circ}, j$ with $i=18.2^{\circ}$ ) give line features that are too strong and too deep.

4. The best fits for the shape and depth of the lines (models $c c$, $h h$ ) indicate higher $M_{1}$ values (e.g. $\sim 0.8 M_{\odot}$ ), but give a continuum which is too hot.

5. The best line + continuum compromise comes from the $b b$ and $g g$ models $\left(M_{1}=0.55 M_{\odot}\right)$ but only when seen at rather high inclination angles, $i=75.5^{\circ}$.

Figure 8 is a plot of the "average" SWP spectrum of 1980 together with the " $g g$ " model $\left(M_{\mathrm{wd}}=0.55 M_{\odot}\right.$, from Wade \& Hubeny 1998) with $i=75.5^{\circ}$.
In conclusion, the fits to the Wade \& Hubeny models (1998), give a range of acceptable values that are "compatible" with the physical parameters of HR Del. Certainly, models with $M_{1}$ larger than $0.8 M_{\odot}$, (too steep continuum, too wide lines) and models with $M_{1}$ lower than $0.55 M_{\odot}$ (too deep lines) are ruled out. A refinement of the grids to include additional intermediate values would be effective in setting tighter constrains to the parameters. However, in any case, as we shall see below, the observed continuum luminosity would need to be explained by a considerably higher accretion rate than the $10^{-8} M_{\odot} \mathrm{yr}^{-1}$ limit of the Wade \& Hubeny (1998) models.

\subsection{The $\lambda \lambda 1180-3250 \AA$ continuum distribution}

In a different approach to the study of the UV continuum we can assume that its distribution can be represented by a blackbody (BB) and/or a power-law (PL) and we can try to determine the parameters that provide the best fit to the UV data.

The UV continuum is clearly defined longwards of $\lambda 1400 \AA$ where only a few and well known spectral lines are present. For a correct definition of the continuum below $\lambda 1400 \AA$ where the continuum position is more uncertain we have been helped by a preliminary identification of the spectral features (most of them being absorption lines, as mentioned in Sect. 3). Also, an impersonal method (the icfit task in IRAF) has yield a similar result. A curve fitting to this continuum has been made using several data analysis packages (gnuplot, Dataplot, Grace) but especially the IRAF stsdas.analysis.fitting.nfit1d application. This code fits 1-dimensional non-linear functions (BB, PL, and combinations of them) to the data. The non-linear fitting can be performed by any of two algorithms, either one of which can be used to minimize chi-squared: the downhill simplex ("amoeba") or the 


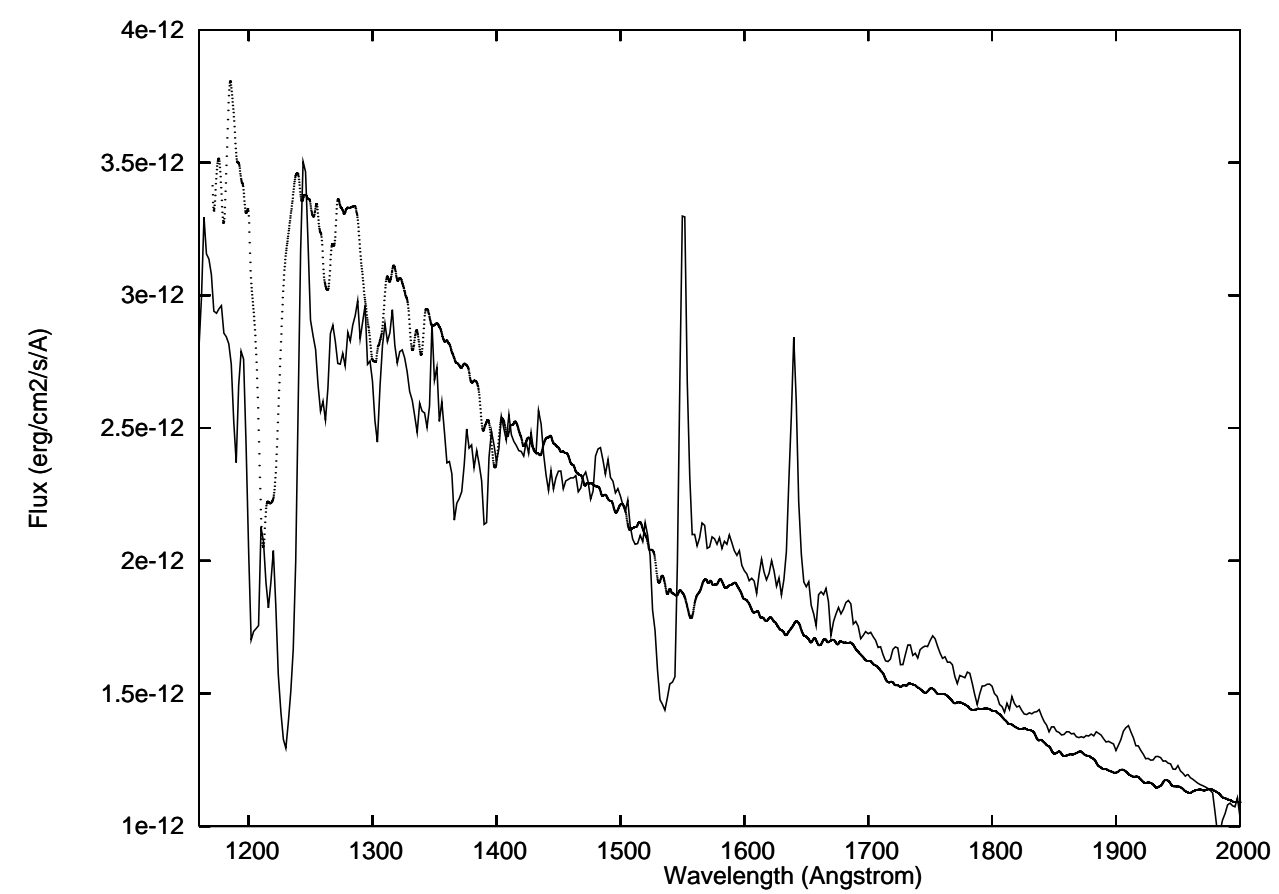

Fig. 8. The reddening corrected "average" far-UV spectrum in 1980 (continuous line) against the " $g$ " model of Wade \& Hubeny (1998) for $i=75.5^{\circ}$ (dots). The ordinate scale of the model has been vertically shifted in order to properly fit the data.

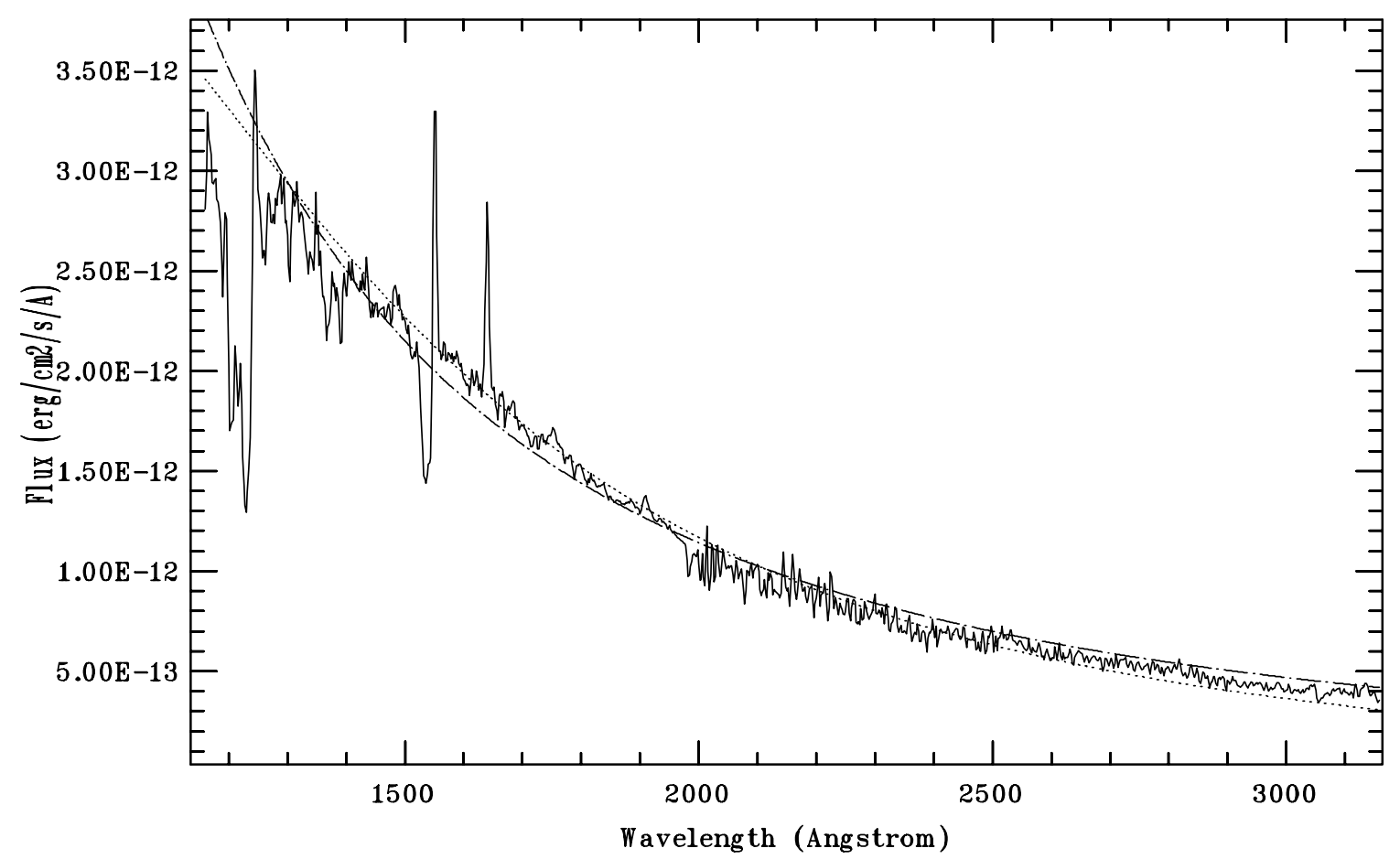

Fig. 9. The "average" 1980-1988 UV spectrum (see Fig. 1) after correction for $E_{B-V}=0.16$. A $33900 \mathrm{~K}$ black-body curve (dots) and a power-law curve with index $\alpha=-2.2$ (dot-dashed line) are superposed.

Levenberg-Marquardt algorithm. The fitting algorithm needs initial guesses for the function coefficients (parameters) that can be entered interactively. The best single curve fit to the (impersonal) UV continuum distribution of HR Del corresponds to a BB with $T \sim 33900 \mathrm{~K}$, while a BB with a slightly lower $T$ is still the best fit to the "hand traced" continuum. A power-law distribution with $F_{\lambda}=2.0 \times 10^{-5} \times \lambda^{-2.20}\left(\mathrm{erg} \mathrm{cm}^{-2} \mathrm{~s}^{-1} \AA^{-1}\right)$ is also a good approximation with a small uncertainty of \pm 0.05 in the index, depending again on the assumed continuum. In any case, the PL fitting gives a spectral index $(\alpha=2.20)$ that is near the Lynden-Bell law for a standard disk $(\alpha=-2.33)$ as already found by Friedjung et al. (1982) from the early IUE spectra. A composite (BB+PL) fitting is obtained with a BB with $35100 \mathrm{~K}$ and a power-law with index -1.84 , but 
does not show any significant improvement to the good fit provided by the single BB at $33900 \mathrm{~K}$. It should be pointed out that there is neither any indication nor any requirement for a high $T$ component: we have tried to "force" such a presence by making such a guess for the initial coefficients but the algorithm has always pointed toward lower temperatures.

As a matter of fact, the best fit to the continuum, the one with the lowest residuals, comes from the combination of two BBs, one with $T=34700 \mathrm{~K}$ and the other with $T=5200 \mathrm{~K}$. The contribution of the latter starts at wavelengths larger than about $\lambda 2600 \AA$ and its extrapolation becomes gradually dominant toward the optical range. It is tempting, "prima facie", to interpret this behavior in terms of a two component continuum with the hot component (the AD) that dominate the UV, and a cool component, presumably the companion star, that is associated with the optical magnitude $m_{v}=12$. However, simple calculations show that an object with $T=$ $5200 \mathrm{~K}$ (probably a $\mathrm{K} 0 \mathrm{~V}$ star), and $R=0.55 R_{\odot}$ at the distance of $850 \mathrm{pc}$ would produce a flux of about $1.0 \times$ $10^{-15} \mathrm{erg} \mathrm{cm}^{-2} \mathrm{~s}^{-1} \AA^{-1}$ at $\lambda 5480 \AA$, the effective wavelength of the $\mathrm{V}$ filter for a low temperature star. This flux can be converted to $\mathrm{m}_{v}$ using the zero mag absolute calibration (Gray 1992)

$\log F_{\lambda}=-0.4 \times V-8.45 \operatorname{erg~cm}^{-2} \mathrm{~s}^{-1} \AA^{-1}$.

This yields $m_{v}=16.0$, that is, $m_{v}=16.5$ after "reddening" it by $A_{V}=0.50$. The same conclusion can be directly obtained by estimating the apparent visual magnitude of a K0 V star $\left(M_{v}=\right.$ $+5.9)$ if $d=850 \mathrm{pc}$ and $A_{v}=0.50 \mathrm{mag}$ : the resulting value is close to $m_{v}=16$. In order to produce $m_{v} \sim 12$ the alleged cool component with $T=5200 \mathrm{~K}$ requires a radius of about $6 R_{\odot}$, a requirement that would imply the presence of a very evolved secondary, not compatible with the well defined orbital period $P=0.214165$. Therefore, we consider the requirement for a cool component just as an analysis "artifact" that might simply arise from the fact that the continuum distribution curve is not a perfect black body.

In conclusion: a BB with $T=33900 \mathrm{~K}$, or alternatively a power-law with $\alpha=-2.20$ represent a good fit to the observed UV continuum distribution. Other fits, although apparently more accurate, lack on a physical basis. We take the temperature $T=33900 \mathrm{~K}$ as indicative of the "mean" accretion disk temperature.

It is remarkable that the extrapolations at $\lambda 5450 \AA$, (the $\lambda_{\text {eff }}$ of the $V$-band for a hot star) of either of these single function distributions that best fit the observed UV continuum (black-body, power-law) yield optical fluxes $(4.7 \times$ $10^{-14} \mathrm{erg} \mathrm{cm}^{-2} \mathrm{~s}^{-1} \AA^{-1}$ and $11.8 \times 10^{-14} \mathrm{erg} \mathrm{cm}^{-2} \mathrm{~s}^{-1} \AA^{-1}$ ) that after conversion to magnitudes and proper "reddening", as described previously, give $m_{v} \sim 12.7$ and $m_{v} \sim 11.7$ respectively.

Therefore, the "quiescent" optical magnitude at $12^{\mathrm{m}}$ corresponds with the "tail" of the UV continuum distribution, that is the tail of the "hot" source.

The optical flux $\left(5 \times 10^{-14} \mathrm{erg} \mathrm{cm}^{-2} \mathrm{~s}^{-1} \AA^{-1}\right)$ found by Ringwald et al. (1996) is close to our UV extrapolated values. The presence of optical nebular lines and the hot continuum reported in the same study confirm the persistence of the hot source observed with IUE.
Figure 9 is a plot of the de-reddened "average" spectrum together with the single power-law and single BB fittings. Toward longer wavelengths the single black body distribution with $T \sim 33900 \mathrm{~K}$ falls slightly below the well defined continuum, while the power-law falls above it.

\section{4. $L_{U V}, M_{v}$ and $\dot{M}$}

The reddening corrected UV-integrated $(\lambda \lambda 1160-3250 \AA)$ continuum flux is of $2.5 \times 10^{-9} \mathrm{erg} \mathrm{cm}^{-2} \mathrm{~s}^{-1}$. If $d=850 \mathrm{pc}$ then the reddening corrected total UV luminosity is $2.2 \times 10^{35} \mathrm{erg} \mathrm{s}^{-1}$ that is, $L_{\mathrm{UV}} \sim 56 L_{\odot}$.

We recall that in the best observed old novae $L_{\mathrm{UV}}$ is in the range 1-20 $L_{\odot}$ (Gilmozzi et al. 1994; Selvelli et al. 2003) the highest values being associated with systems seen near poleon, while for systems at intermediate inclination, like HR Del, a typical value is $\sim 5 L_{\odot}$. See also Friedjung \& Selvelli (2001) for other considerations on the peculiar luminosity of HR Del. Therefore, HR Del appears as the brightest member in the UV among ex novae and is challenged only by the two recurrent novae T Cr B and T Pyx, at $L_{\mathrm{UV}} \sim 50 L_{\odot}$ (Selvelli et al. 1992) and $L_{\mathrm{UV}} \sim 98 L_{\odot}$ (Gilmozzi et al. 2003), respectively. Incidentally, we point out that the overall (line and continuum) UV spectral appearance of HR Del would be quite similar to that of the recurrent nova T Pyx, were it not for the absence in the latter star of the P Cyg absorption components in the NV and CIV resonance lines (See also Sect. 6.7).

In the following we confidently assume that the observed UV luminosity comes from an accretion disk heated by viscous dissipation of gravitational energy. This sounds reasonable in view of the general behavior in other similar objects, old novae and nova-like stars, and is supported by the fact that the UV continuum distribution is close to that expected for a "standard" optically thick AD, as well as that predicted by an early simplistic model of an irradiated AD (Friedjung 1985). In Sect. 6.6 we consider also some problems that arise if we assume the presence of a hot, bloated WD as the main source for the UV continuum.

From $m_{v}=12$, assuming $d=850 \mathrm{pc}$, and $A_{V}=0.50$ $\left(E_{B-V}=0.16\right)$ we obtain $M_{v}=+1.85$ for the "apparent" absolute magnitude. Supposing that the visual radiation comes from a non-irradiated disk we correct for the inclination $\left(i \sim 40^{\circ}\right)$ by a factor $\Delta M_{v}=0.45$, an average from the Paczynski \& Schwarzenberg-Czerny (1980), Warner (1986) and Webbink et al. (1987) relations, to obtain an absolute magnitude avertaged over all directions of $M_{v}=+2.30$. This $V$ is brighter by about 1.7 mag than the mean absolute magnitude of nova remnants in the same speed class, whose average value is centered on $M_{v}=+4.0$ (See Fig. 2.20 and Table 4.6 of Warner 1995). This $V$ is also brighter by about 2.4 mag than the mean absolute magnitude of novae at minimum, as obtained from the values given in Table 6 of Downes \& Duerbeck (2000). In this list, only one object (BT Mon) appears brighter than HR Del, but, being a high inclination system, its $\mathrm{V}$ value could be affected by a substantial uncertainty in the correction.

The "pole-on" $(i=0)$ absolute visual magnitude of HR Del obtained by the same correction method (but with a negative 
value for a disk viewed "pole-on") is close to $M_{v}=+1.35$. This value is also brighter by about $1.3 \mathrm{mag}$ than the average value for nova remnants seen "pole-on" (See Fig 2.20 of Warner 1995).

Therefore, HR Del at the "quiescent" $m_{v}=12$ is one of the brightest nova remnants also in the optical range. This result is not very surprising if one considers the peculiar UV luminosity of HR Del and the fact that, as mentioned before, $m_{v}$ comes from the same source.

We emphasize that the corrections for inclination mentioned above are valid only if the observed continuum comes from a disk that is heated by viscous dissipation of gravitational energy. The luminosity of a bare WD clearly needs no inclination correction. Lapidus \& Sunyaev (1985) discuss the case of an irradiated disk, giving an inclination correction, which leads to the increase of the calculated bolometric luminosity of the irradiating central object by a factor near 3.6.

We recall that Warner (1987) has given $M_{v} \sim+3.9$ (and also +4.2) for HR Del as an ex-nova, but he has assumed $d=$ $285 \mathrm{pc}$, a value that seems difficult to reconcile with all other estimates.

In principle, the mass accretion rate $\dot{M}$ in a viscously heated disk can be estimated from a comparison between the observed spectral distribution and that of proper models. However, the number of parameters in any disk model is quite large and the various methods of fitting the data to the models do not generally provide unequivocal results. On the other hand, if the total disk luminosity and the mass of the WD are known, $\dot{M}$ can be directly calculated from the relation

$\dot{M}=\frac{2 R_{1} L_{\mathrm{disk}}}{G M_{1}}$

In this case, the estimate of $\dot{M}$ is not model dependent but requires the knowledge of the bolometric accretion luminosity $L_{\text {disk }}$ and of $M_{1}$. The observed $L_{\mathrm{UV}}$ can provide a first estimate of $L_{\mathrm{disk}}, L_{\mathrm{UV}}$, being a lower limit to the bolometric disk luminosity $L_{\text {disk }}$.

We recall that most of the accretion luminosity is emitted in the IUE-UV range: radiation at wavelengths shorter of $\operatorname{Ly} \alpha$ is strongly absorbed and the energy is redistributed toward longer wavelengths (Nofar et al. 1992). If the mean accretion disk temperature is close to $33900 \mathrm{~K}$, with the assumption that the distribution is close to that of a $\mathrm{BB}$, one can easily see that the peak of the distribution falls near $\lambda 850 \AA$ and that about half of the total power is emitted shortwards of about $\lambda 1200 \AA$. It seems therefore justified to assume $L_{\text {disk }} \sim 2 L_{\mathrm{UV}}$, that is, $L_{\text {disk }} \sim 112 L_{\odot}$. Also, a detailed examination of the Wade \& Hubeny (1998) grid of model spectra for the relevant cases shows that approximately only one half of the total continuum energy is emitted shortwards of $\lambda 1160 \AA$.

If we adopt $L_{\text {disk }} \sim 112 L_{\odot}$, together with $M_{1} \sim 0.65 M_{\odot}$ and $R_{1} \sim 0.0125 R_{\odot}$ we obtain that $\dot{M} \sim 1.4 \times 10^{-7} M_{\odot} \mathrm{yr}^{-1}$.

An independent determination of the mass accretion rate can be obtained through the $\dot{M}$-HeII $1640 \AA$ luminosity relation given in Table 2 of Patterson \& Raymond (1985). The average (de-reddened) flux on earth in the HeII 1640 line is of $7.0 \times 10^{-12} \mathrm{erg} \mathrm{cm}^{-2} \mathrm{~s}^{-1}$ and the corresponding luminosity is $6.1 \times 10^{32} \mathrm{erg} \mathrm{s}^{-1}$. If $M_{1}=0.7 M_{\odot}$ this corresponds to a mass accretion rate $\dot{M} \sim 10^{19} \mathrm{gr} \mathrm{s}^{-1}$, that is $\dot{M} \sim 1.4 \times 10^{-7} M_{\odot} \mathrm{yr}^{-1}$, in excellent (if not surprising) agreement with the estimate based on the UV continuum.

The mass accretion rate $\dot{M}$ can be also estimated from the "average" $M_{v}$. using the relation of Webbink et al. (1987):

$\dot{M}=\frac{10^{0.6\left(-9.48-M_{v}\right)}}{M_{1}}$.

Substitution of the specific values for HR Del $\left(M_{1}=0.65 M_{1}\right.$, $\left.M_{v}=+2.30\right)$ gives $\dot{M}=1.31 \times 10^{-7} M_{\odot} \mathrm{yr}^{-1}$. We point out that the $\dot{M}$ obtained by this method comes from the adoption of a $M_{v}$ value that corresponds to an "average" disk $\left(i \sim 58^{\circ}\right)$.

The remarkable fact that three different methods yield very similar $\dot{M}$ values supports of the reliability of the results (and of the methods).

An estimate of the mean size of the emitting surface can be obtained using the "mean" disk temperature $T \sim 33900 \mathrm{~K}$ (see Sect. 6.3) and the total disk luminosity $L_{\text {disk }} \sim 2 \times L_{\mathrm{UV}} \sim$ $112 L_{\odot}$. We obtain $R \sim 0.36 R_{\odot}$, in good agreement with the expected size of an accretion disk in a cataclysmic variable.

It must be stressed that all the quite high $\dot{M}$ values obtained above come from the adoption of a rather conservative (low) value for the distance. In order to obtain values for $L_{\mathrm{UV}}$, $M_{v}$ and $\dot{M}$ close to those found in other ex-novae one should adopt a much lower value for the distance $(d \leq 400 \mathrm{pc})$, but this is sharp contrast with all more recent estimates that give $d \sim 1 \mathrm{kpc}$.

Using the values for $\dot{M}, M_{1}$, and $R_{1}$ derived previously, we can estimate the value of the "maximum" disk temperature from the common relation

$T_{\mathrm{disk}}^{\max }=0.488\left(\frac{3 G M_{1} \dot{M}}{8 \pi \sigma R_{1}{ }^{3}}\right)^{1 / 4}$.

We obtain $T_{\text {disk }}^{\max } \sim 1.08 \times 10^{5} \mathrm{~K}$, to be compared with the maximum disk temperature in the relevant models of Wade \& Hubeny (1998) that ranges from $T \sim 39110 \mathrm{~K}$ (model $j j$ ) to $T \sim 69560 \mathrm{~K}$ (model $h h$ ) and with the "mean" disk temperature $T=33900 \mathrm{~K}$ estimated in the previous chapter.

\subsection{The pre-nova and the ex-nova}

The comparison between the pre-nova and the post-nova magnitudes has been the focus of various investigations that have led to non-unequivocal results and interpretations.

Payne-Gaposchkin (1957) from a study of 12 novae concluded that the pre-nova and post-nova brightnesses (years after outburst) are more or less the same. Robinson (1975), from a slightly larger sample, fundamentally confirmed that the mean magnitudes of the pre-nova and the post-nova are essentially the same, but also reported for many novae a moderate rise in brightness in the 1-15 years prior to the eruption. Shara et al. (1986), in the context of the "hibernation" theory, claimed that pre novae become "bright" (at $M_{v}$ near +4.0 , the average absolute magnitude of novae before and after eruption) a few decades before the eruption, and old novae remain bright at near the same $M_{v}$ for many decades after outburst. The high values observed in $\mathrm{CN}$ before and after eruption are allegedly 
produced by the resumption of contact of the red dwarf with its Roche lobe (after the drain of angular momentum from the system by gravitational radiation or by magnetic braking by a stellar wind) and by the irradiation of the secondary, respectively. The hibernation theory predicts an order of magnitude decrease in the quiescent luminosity over a long timescale (several decades or a century; Kovetz et al. 1988) and therefore good data on the secular brightness evolution of post-novae are required to test the theory. Vogt (1990) in a study of 97 well observed galactic novae found that during the first 130 years after the eruption galactic novae show a slow decrease in brightness with a rate of 2.1 mag per century and interpreted this as evidence in support of hibernation. Duerbeck (1992), from a detailed study of a limited number of well selected post-novae, based on observations obtained at least $20 \mathrm{yr}$ after outburst, found instead a decline rate of $10.4 \mathrm{mmag} /$ year and a behavior compatible with hibernation

The situation seems however controversial: Weight et al. (1994) examining near IR photometry for a number of exnovae found no correlation between $\dot{M}$ and time since outburst. Recently, Duerbeck (1995) has reviewed the general assumption that $m$ (pre-nova) $=m$ (post-nova) and found that most novae, some years after outburst are about $2^{m}$ brighter than before outburst, and decline gradually to pre-outburst brightness in about 10-30 years of exponential decay.

In the case of HR Del, we recall that Stephenson (1967) found $m_{v}$ about 12 for the pre-nova magnitude and that Wenzel (1967), from the inspection of Sonnenberg plates taken between 1928 and 1966 found the pre-nova to be only slightly variable around a mean photographic magnitude of 11.9. Van den Bergh \& Racine (1967) also found evidence of small variations in brightness from a comparison between PSS plates obtained in 1951 and 1953 and pointed out that the pre-nova was very blue.

HR Del is one of the few novae for which pre-nova spectra are available: Stephenson (1967) classified the pre-nova continuum (seven years prior to outburst) to be that of an $\mathrm{O}$ or early B star while Hutchings (1968) estimated the temperature of the pre-nova star to be $\sim 32000 \mathrm{~K}$. Barnes \& Evans (1970) from photographic photometry on Palomar Sky Survey plates found $(B-V)=-0$. 18 for the pre-nova in 1951. After correction for $E_{B-V}=0.16$, this gives $(B-V)_{\mathrm{o}}=-0.34$ in good agreement with the value expected from an object with $T \sim 33000 \mathrm{~K}$. Also, Seitter (1990), from an uncalibrated low-resolution prenova spectrum of HR Del found evidence of high temperature continuum.

After the outburst of 1967 the extra slow decline in $m_{v}$ of HR Del lasted for about 15 years with an asymptotic approach toward the pre-outburst value $\left(m_{v}=12\right)$ that was reached around 1981-1982. Thus, most IUE observations were taken after the return of the nova to the pre-outburst $V$-magnitude.

In conclusion, for many years before the 1967 outburst HR Del has been at the same $m_{v}$ and $T$ values $\left(m_{v} \sim 12.0\right.$ and $T=33000 \mathrm{~K}$ ) as during the post-nova stages corresponding to most IUE observations.

Recalling (Sect. 6.3) that the "quiescent" magnitude at $m_{v}=12$ comes from the "tail" (extrapolation to $\lambda 5450 \AA$ ) of the observed hot UV continuum, which is close to both a power-law and a $32900 \mathrm{~K}$ black-body, (and is not due to the cool companion which can account for only a small fraction of the required $V$ flux) it follows that the star during the pre-nova stage at $12^{\mathrm{m}}$ had necessarily the high $T, L_{\mathrm{UV}}, M_{v}$ and deduced $\dot{M}$ values found in the present study.

We point out these peculiar aspects in the behavior of HR Del:

1. As a post-nova, HR Del at $L_{\mathrm{UV}}=56 L_{\odot}$ and $M_{v}=2.30$ is brighter than its colleagues both in the optical and in the UV. One could expect this behavior if the post nova had remained in an "excited state" at, say, $m_{v}=11.6$, because of the extreme slowness of the outburst phases and of a corresponding extra slow decline toward the pre-outburst brightness. Instead, this "bright" state corresponds to the return to the pre-outburst magnitude.

2. As demonstrated previously, during at least 70 years before the 1967 outburst at $m_{v} \sim 12$, HR Del was necessarily at the same high $T, L_{\mathrm{UV}}$, and deduced $\dot{M}$, as derived from the IUE and other recent observations. This sets a time constraint to a possible pre-outburst onset of high $\dot{M}$.

3. Apparently, there is no indication of any further decline in optical brightness during the last 18 years, that is, after the return to $m_{v} \sim 12.0$.

We cannot but point out that, in the context of the "hibernation" theory, it is difficult to understand how two different processes (the contact induced $\dot{M}$ in pre-outburst and the irradiation induced $\dot{M}$ in the post-outburst) could have led to the same $\dot{M}$ values, as to produce the same pre-and post-outburst magnitudes. The process of irradiation of the secondary should have been especially important for HR Del because of the extra-slow character of the outburst that has kept both the WD and the companion exposed for a long time to the hard radiation of the nova.

In other words it is not clear how, many years before outburst HR Del managed to be at near the same high values for $\dot{M}$, $L, T$ as in the post-nova stage.

It is worth recalling that the magnitude range $A=$ $m_{v}$ (outburst) $-m_{v}$ (post-nova) of HR Del $(\sim 8 \mathrm{mag})$ is within the average behavior (Warner 1995) of other members of the extra slow class. This parameter however depends on the difference between the pre-outburst and the post-outburst values, and not on the absolute values.

\subsection{A still burning white dwarf?}

Hydrodynamic models of nova outburst predict that, after the onset of the outburst, hydrogen nuclear burning should continue on the surface of the WD for as long as 100 years, with a strong inverse dependence on the mass of the white dwarf. Real novae instead seem quite inpatient and are able to shorten considerably this phase through some not well established mechanism (enhanced mass-loss via radiation pressure-driven winds, common envelope ejection, magnetic fields, etc). Combined UV + soft X-ray observations (Gonzalez-Riestra et al. 1998; Vanlandingham et al. 2001) have indeed shown that most novae decline in bolometric luminosity within a few years from the outburst. 
Sion \& Starrfield (1994) have presented theoretical results on the processes of compressional heating, low-level hydrogen burning, but not accretion on very hot, low-mass WD, and found that these processes power the models. The luminosities are a few times $10^{37} \mathrm{erg} \mathrm{s}^{-1}$ at certain epochs, being much less (e.g. a few times $10^{35} \mathrm{erg} \mathrm{s}^{-1}$ ) at other times. They suggest that these models could be relevant to the class of ultrasoft X-ray sources and related objects.

In this framework, it is conceivable that some aspects in the observed behavior of HR Del, e.g. the observed high UV luminosity and the strong P Cyg profiles, indicative of outflow, could be interpreted as evidence that continuous hydrogen burning is taking place on the surface envelope of a low mass white dwarf. Also, the conservative estimate for the absolute visual magnitude of HR Del in quiescence $\left(M_{v}=+2.30\right.$, Sect. 6.4) would place it in the region of the supersoft binaries according to Fig. 2 of Patterson (1998).

However, both the slope of the continuum (that is indicative of a temperature of the order of $33900 \mathrm{~K}$ ) and the character of the line spectrum (especially the relative intensities of the CIV $1550 \AA$ and HeII $1640 \AA$ emission lines, and the weakness of the NV $1240 \AA$ line) are hardly compatible with the presence of a very hot WD with $T \sim 2 \times 10^{5} \mathrm{~K}$, as expected in this case, unless some kind of reprocessing of the "hard" radiation takes place, e.g. in the irradiation of the accretion disk. The presence of a compact and very hot continuum source, whose contribution toward the UV-optical regions is relatively small, could also be invoked but in any case the absence or weakness of emission lines of high excitation is disturbing. Also, the observed absorption lines of SiII at $1260 \AA$, and CII at $1335 \AA$, etc., are indicative of stellar spectra about B2-B7 with $T>20000 \mathrm{~K}$ and $T<32000 \mathrm{~K}$, in fair agreement with the $T$ from the continuum, and indicative of a multi- $T$ structure, as expected in an $\mathrm{AD}$.

Another argument against the hypothesis of a still burning WD lies in the difficulty of reconciling the hypothesis of an enduring phase of continuous burning on the WD surface with the fact that the system (ex-nova) is exactly at the same prenova optical magnitude. This would imply that the pre-outburst "quiescent" magnitude at $m_{v} \sim 12$ (almost constant in accordance with all "historical" data available) (line and continuum) a phase of stable burning which might even have endured since the previous outburst.

\subsection{The wind features and their origin}

The absorption component in the CIV $1550 \AA$ A doublet reaches $v_{\text {edge }} \sim 5000 \mathrm{~km} \mathrm{~s}^{-1}$ and its shape suggests the presence of two structured components displaying their maximum depth near the line center. A similar behavior is displayed by the NV $1240 \AA$ doublet, but because of the proximity of $\mathrm{Ly}_{\alpha}$ a precise measurement is difficult. It should be noted that this high $v_{\text {edge }}$ value contrasts with the moderate outflow velocities recorded at the time of the outburst when most spectroscopic observations showed velocities in the range from -200 to $-700 \mathrm{~km} \mathrm{~s}^{-1}$ (Hutchings 1968). In any case the presence for at least 25 years after outburst of strong wind features in the line spectrum of HR Del is surprising. In the first IUE spectra of 1979 they were interpreted in the framework of a continuing mass outflow in the outburst of a very slow nova (Hutchings 1979). However, the absorption profiles were still present, although slightly weaker, in the last IUE spectra of 1992 and presumably they still are.

The phenomenology of the short-time variations of the wind (as judged from the behavior of the CIV and NV absorption components) indicates the presence of an inhomogeneous (structured) outflow with irregular variations on short timescale and the sudden ejection of puffs of optically thick material. This behavior is similar to that observed in other CVs and especially in V 603 Aql. In this context, we recall that Friedjung et al. (1997), from a GHRS study of V 603 Aql, suggested that the emission and absorption components of the $\mathrm{P}$ Cyg profiles originated from two separate physical regions, i.e. a chromosphere corona which surrounds the disk, and a conical-shaped wind region, nearly perpendicular to the disk itself. In a follow-up IUE study on the variations in the wind features of V $603 \mathrm{Aql}$, Selvelli et al. (1998) pointed out that the outflow should take place through the sudden ejection of puffs of optically thick material, with timescales on the order of tens of minutes or less. No definite periodicities were associated with the presence of the wind nor any correlation between the absorption features and the UV flux modulation detected. A similar conclusion was reached by Prinja et al. (2000) from GHRS data of V 603 Aql taken with high time resolution: they observed variability on timescales of minutes and suggested an empirical picture of stochastically variables structures in the outflow with no evidence for any cyclic modulation in the absorption components properties.

The similarity in the class of wind phenomena between HR Del and V 603 Aql suggest that the same basic mechanism, that is, a collimated outflow (conical wind) from the inner accretion disk region is present in both stars. The absence of any correlation between the absorption components and the changes in the UV flux or the EUV flux (from the HeII $1640 \AA$ line) should set some definite constrains on the physical interpretation. Prinja et al. (2000) concluded that the irregular absorption episodes of V 603 Aql defied a clear physical interpretation, and a similar conclusion, regrettably, seems valid for HR Del also: the origin and region of formation of the wind features are not clear. We recall that the presence of short time variations in the profiles of the resonance lines has been reported also for several other CVs systems not belonging to the $\mathrm{CN}$ subclass.

In the framework of the hypothesis of a bi-conical outflow, after correction for the inclination $\left(i \sim 40^{\circ}\right)$, a quite high value for the intrinsic $v_{\text {edge }}$, on the order of $7000 \mathrm{~km} \mathrm{~s}^{-1}$, is obtained. We recall that Hutchings (1980) already noted that in HR Del the terminal velocity was higher that in other stars except perhaps some WR stars. We recall also that a very low system inclination is not compatible with the other system parameters and that a lower limit for $i$ is about $35^{\circ}$. In other $\mathrm{CVs}$, the observed terminal $v$ is generally comparable to the escape velocity from the primary. For HR Del, such high value would indicate a massive WD, but the outburst behavior and data on the system suggest that the WD in HR Del is rather 


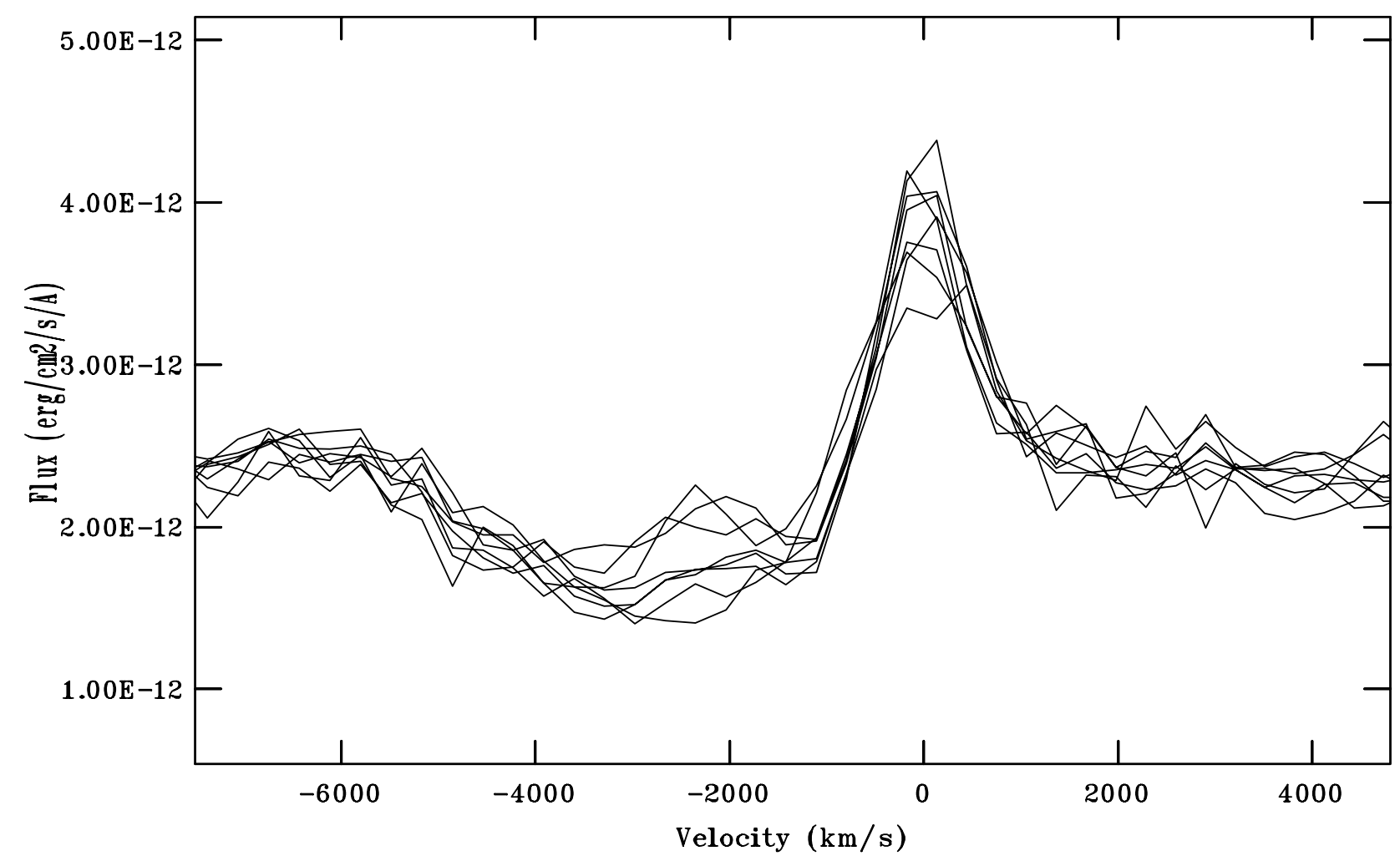

Fig. 10. The short-timescale changes in the wind absorption features of the CIV $1550 \AA$ A resonance doublet. The spectra belong to the sequence of 21 Aug., 1980 and the time separation between two successive SWP exposures is about $49 \mathrm{~min}$.

undermassive compared with other $\mathrm{CN}\left(M_{\mathrm{wd}} \sim 0.65 M_{\odot}\right)$ and a lower $v_{\text {esc }}$ is expected. It is worth to point out that in V $603 \mathrm{Aql}$, where, allegedly, a more massive WD is present, the observed $v_{\text {edge }}$ reaches a value on the order of $-2700 \mathrm{~km} \mathrm{~s}^{-1}$, (that is, $v_{\text {edge }} \sim-2850 \mathrm{~km} \mathrm{~s}^{-1}$, after correction for the low $i$ ).

The problem of an exceeding high value for $v_{\text {edge }}$ could be alleviated only if the outflow took place in a spherical geometry. However, an outflow from the whole WD is difficult to envisage without invoking some kind of persistence of TNR activity on the surface of a bloated white dwarf. We have above mentioned some difficulties connected with this hypothesis, at least if it is supposed that no accretion disk is present. In any case, the apparent similarity in the phenomena of the short timescale variations in HR Del with those in V 603 and other CVs may favor an interpretion in terms of phenomena taking place in a bi-conical AD geometry.

It seems quite obvious to associate the presence of the strong P Cyg profiles with that of a strong UV luminosity. In this case the high velocity P Cyg absorption components could be due to a wind driven by a radiation force in the lines. In any case, it seems to us that the radiation force alone cannot account for the observed wind properties of HR Del: the recurrent nova T Pyx has nearly the same UV continuum shape and an higher UV luminosity than HR Del but shows no evidence of any absorption component despite the fact of being observed at a more favorable (lower) inclination angle $\left(i \sim 17^{\circ}\right)$. A nonradiative factor such as the presence of a strong magnetic field could be invoked (see Proga 2000; Hartley et al. 2002) but no evidence for it has been found so far in HR Del. In addition, the presence of puffs and structures in the wind remains a challenge to our understanding of this class of phenomena.

Downes \& Duerbeck (2000), in measuring the distance of HR Del with the method of the expansion parallax, have found some evidence of acceleration in the shell. They have interpreted this result as an indication of the presence of a fast wind from the stellar remnant, as supported by the P Cyg profiles detected in the first IUE spectra (Krautter et al. 1981). On the basis of our previous considerations it may seem clear that the wind geometry is not spherical, and that if the outflow axis is perpendicular to the disk, acceleration is expected only in the polar caps. In turn, this would enhance the prolateness of the ejecta and this could provide an alternative interpretation for the prolateness of the remnant found by Slavin et al. (1994) and attributed by them to the common envelope phase during the nova outburst. In this respect see also O'Brien et al. (2002).

In an alternative view with respect to the origin of the wind, we recall that Hachisu et al. (1996) have found that the white dwarf begins to blow (bi-conical) optically thick winds when the mass accretion rate $\dot{M}$ exceeds the critical rate $\left(\sim 1.9 \times 10^{-7} M_{\odot} \mathrm{yr}^{-1}\right.$ for a $0.7 M_{\odot} \mathrm{WD}$, see also Fig. 2 in Hachisu \& Kato 2001) at which steady shell-burning can process the accreted hydrogen into helium. At such rates the WD envelope is supposed to expand to $R_{\mathrm{ph}} \sim 0.1 R_{\odot}$ while $T_{\mathrm{ph}}$ decreases below $3.2 \times 10^{5} \mathrm{~K}$. If $\dot{M}$ decreases below the critical value, optically thick winds stop. If the mass transfer rate further decreases below about $0.5 M_{\text {crit }}$, $\mathrm{H}$ shell burning becomes unstable to trigger weak shell flashes. We wonder whether the strong wind observed in HR Del can somehow be associated 
with this model: on the one hand the model luminosities and temperatures are exceedingly high as compared with those found in the present study, on the other hand the critical accretion rate from Hachisu et al. (1996) is very close to the $\dot{M}$ value derived in Sect. 6.4.

\section{Conclusions}

There are aspects in the UV and optical behavior of HR Del that are intriguing and makes it peculiar among ex-novae:

1. In spite of of a conservatively low value assumed for the distance, HR Del appears to be one of the brightest remnants (if not the brightest one) among classical novae both in the $\mathrm{UV},\left(L_{\mathrm{UV}} \geq 56 L_{\odot}\right)$, and in the optical, $\left(M_{v}=+2.30\right)$, assuming that radiation comes from a non-irradiated accretion disk. This peculiar brightness could be attributed to a form of persisting post-outburst activity (low level thermonuclear burning?) associated with the extra-slow character of the nova, were it not that most IUE observations were made near or after its return, around 1981, to the "prenova quiescent" magnitude at $m_{v} \sim 12$. Over the 13 years of the IUE observations the UV continuum has declined by a factor less than 1.2. However, the much larger decrease of the fluxes in the emission lines of highly ionized atoms suggests cooling of the source of ionizing radiation, in the framework of photoionization.

2. Since the optical "quiescent" magnitude at $12^{\mathrm{m}}$ has its origin in the hot component (the "tail" of the UV continuum) and not from the late-type companion star. it follows that the pre-nova, observed at $m_{v}=12$ for at least 70 years prior to outburst, was at the same (high) $T, L$, and $\dot{M}$ values as directly derived for the ex-nova in the present study. It is not clear why and how the pre-nova was so bright, and we wonder whether both the pre-nova and ex-nova minima at $m_{v}=12$ are real "minima" or correspond to some kind of long time-scale nuclear burning as predicted by the low WD mass models of Sion \& Starrfield (1994).

3. The presence of strong P Cyg profiles in the CIV and NV resonance lines with an observed $v_{\text {edge }} \sim-5000 \mathrm{~km} \mathrm{~s}^{-1}$ is remarkable. This behavior is intriguing because HR Del is the only ex-nova that permanently displays such strong features and because in other CVs the P Cyg profiles give lower velocities and are generally observed in low inclination systems only. After correction for the inclination $i \sim 40^{\circ}$ the situation becomes worse and a disturbingly high $v_{\text {out }} \sim-7000 \mathrm{~km} \mathrm{~s}^{-1}$ is obtained, a value that is in sharp contrast with the relatively low mass of the white dwarf.

In conclusion: on the basis of the observed behavior of HR Del we have in this paper favored an interpretation in terms of phenomena that are powered by accretion. However, we cannot rule out an alternative interpretation based on phenomena that take place on a still-burning white dwarf (surrounded nevertheless by an accretion disk) that also burns at the same very low rate before a major outburst and perhaps all the time between major outbursts.

Acknowledgements. We gratefully thank Ed Sion for a fruitful discussion during the final stage of this article.

\section{References}

Alcock, G. E. D. 1967, IAU Circ. No. 2022

Anderson, M. 1988, ApJ, 325, 266

Anderson, C. M., \& Gallagher, J. S. 1977, PASP, 89, 264

Barnes, T. G., \& Evans, N. R. 1970, PASP, 82, 889

Bartolini, C., 1969, Mem. Soc. Astron. It., 40, 127

Bruch, A. 1982, PASP, 94, 916

Clemens, J. C., Reid, I. N., Gizis, J. E., \& O’Brien, M. S. 1998, ApJ, 496, 352

Della Valle, M., \& Livio, M. 1995, ApJ, 452, 704

Downes, R. A., \& Duerbeck, H. W. 2000, AJ, 120, 2007

Drechsel, H., Rahe, J., Duerbeck, H. W., Kohoutek, H. W., \& Seitter, W. C. 1977 , A\&AS, 30, 323

Duerbeck, H. W. 1992, MNRAS, 258, 629

Duerbeck, H. W. 1995, in Cataclysmic Variables, ed. A. Bianchini, M. Della Valle, \& M. Orio (Kluwer Acad. Publ.), 39

Duerbeck, H. W., \& Seitter, W. C. 1987, Ap\&SS, 131, 467

Fireman, G. F., \& Imhoff, C. L. 1989, NASA IUE Newslett. 40, 10

Friedjung, M. 1985, A\&A, 146, 366

Friedjung, M. 1992, A\&A, 262, 487

Friedjung, M., Andrillat, Y., \& Puget, P. 1982, A\&A, 114, 351

Friedjung, M., Selvelli, P., \& Cassatella, A. 1997, A\&A, 318, 204

Friedjung, M., \& Selvelli, P. L. 2001, in Evolution of Binary and Multiple Star Systems, ASP Conf. Ser., 229, 363

Gilmozzi, R., Selvelli, P. L., \& Cassatella, A. 1994, Mem. Soc. Astron. It., 65,199

Gilmozzi, R., Selvelli, P. L., \& Cassatella, A. 2003, in preparation

Gonzalez-Riestra, R., Orio, M., \& Gallagher, J. 1998, A\&AS, 129, 23

Gonzalez-Riestra, R., Cassatella, A., Wamsteker, W., A\&A, 373, 730

Gray, D. 1992, The Observation and Analysis of Stellar Photospheres, Cambridge Ap. Ser., 184

Hachisu, I., Kato, M., \& Nomoto, K. 1996, ApJ, 470, L97

Hachisu, I., \& Kato, M. 2001, ApJ, 558, 323

Hartley, L. E., Drew, J. E., Long, K. S., Knigge, C., \& Proga, D. 2002, MNRAS, 332, 127

Hutchings, J. B. 1968, Pub. Dom. Astr. Obs. XII, 397

Hutchings, J. B. 1979, PASP, 91, 661

Hutchings, J. B. 1980, PASP, 92, 458

Kohoutek, L. 1981, MNRAS, 196, 87

Kovetz, A., Prialnik, D., \& Shara, M. M. 1988, ApJ, 325, 828

Krautter, J., Klare, G., Wolf, B., et al. 1981, A\&A, 102, 337

Kuerster, M., \& Barwig, H. 1988, A\&A, 199, 201

Lapidus, I. I., \& Sunyaev, R. A. 1985, MNRAS, 217, 291

Livio, M. 1993, in 22nd Saas-Fee Advanced Course, Interacting Binaries, ed. H. Nussbaumer, \& A. Orr (Berlin: Springer Verlag)

Mannery, E. J. 1970, PASP, 82, 626

Nauenberg, M. 1972, ApJ, 175, 417

O’Brien, T. J., Harman, D. J., \& Bode, M. F. 2002, in Classical Nova Explosions, ed. M. Hernanz, \& J. José, AIP Conf. Proc., 637, 509

Paczynski, B., \& Schwarzenberg-Czerny, A. 1980, Acta Astron. 30, 131

Patterson, J. 1998, PASP, 110, 1132

Patterson, J., \& Raymond, J. C. 1985, ApJ, 292, 550

Payne-Gaposchkin, C. 1957, in The Galactic Novae (North Holland), 28

Perez, M. R. 1991, ESA IUE Newslett. 38, 27

Politano, M., Livio, M., Truran, J. W., \& Webbink, R. F. 1990, in Physics of Classical Novae, ed. A. Cassatella, \& R. Viotti, Lect. Notes Phys. 369, 386

Prinja, R. K., Knigge, C., Ringwald, F. A., \& Wade, R. A. 2000, MNRAS, 318,368

Proga, D. 2000, ApJ, 538, 684

Rafanelli, P., \& Rosino, L. 1978, A\&AS, 331, 337 
Ringwald, F. A., Naylor, T., \& Mukai, K. 1996, MNRAS, 281, 192 Ritter, H., \& Kolb, U. 1998, A\&A, 129, 83

Ritter, H., Politano, M., Livio, M., \& Webbink, R. F. 1991, ApJ, 376, 177

Robinson, E. L. 1975, AJ, 80, 515

Rodriguez-Pascual, P. M., Gonzalez-Riestra, R., Schartel, N., \& Wamsteker, W. 1999, A\&AS, 139, 183

Rosino, L., Bianchini, A., \& Rafanelli, P. 1982, A\&A, 108, 243

Rountree, J., \& Sonneborn, G. 1993, NASA Ref. Publ. 1312

Seitter, W. C. 1990, in Physics of Classical Novae, ed. A. Cassatella, \& R. Viotti, Lect. Notes Phys. 369, 79

Selvelli, P. L., Gilmozzi, R., Friedjung, M., \& Cassatella, A. 1998, ESA SP 413, 435

Selvelli, P. L., Cassatella, A., \& Gilmozzi, R. 1992, ApJ, 393, 289

Selvelli, P. L., Gilmozzi, R., \& Cassatella, A. 2003, in preparation

Shara, M. M., Livio, M., Moffat, A. F. J., \& Orio, M. 1986, ApJ, 311, 163

Sion, E. M., \& Starrfield, S. G. 1994, ApJ, 421, 261

Slavin, A. J., O’Brien, T. J., \& Dunlop, J. S. 1994, MNRAS, 266, L55

Slavin, A. J., O’Brien, T. J., \& Dunlop, J. S. 1995, MNRAS, 276, 353
Smith, D. A., \& Dhillon, V. S. 1998, MNRAS, 301, 767

Solf, J. 1983, ApJ, 273, 647

Stephenson, C. B. 1967, PASP, 79, 584

Terzan, A. 1970, A\&A, 5, 167

Terzan, A., Bally, M., \& Durand, A. 1974, A\&AS, 15, 107

Van den Bergh, S., \& Racine, R. 1967, IBVS, 212

Vanlandingham, K. M., Schwarz, G. J., Shore, S. N., \& Starrfield, S. 2001, ApJ, 121, 1126

Vogt, N. 1990, ApJ, 356, 609

Wade, R. A., \& Hubeny, I. 1998, ApJ, 509, 350

Wallerstein, G. 1968, Astrophys. Lett., 2, 89

Warner, B. 1986, MNRAS, 222, 11

Warner, B. 1987, MNRAS, 227, 23

Warner, B. 1995, in Cataclysmic Variable Stars (Cambridge University Press), 111

Webbink, R. F., Livio, M., Truran, J. W., \& Orio, M. 1987, ApJ, 314, 653

Weight, A., Evans, A., Naylor, T., Wood, J. H., \& Bode, M. F. 1994, MNRAS, 266, 761

Wenzel, W. 1967, IAU Circ., 2026 